\title{
Ring fiber resonators based on fused-fiber grating add-drop filters: application to resonator coupling
}

\author{
John M. Choi, Reginald K. Lee, and Amnon Yariv \\ California Institute of Technology, M/S 136-93, 1200 East California Boulevard, Pasadena, California 91125
}

Received April 22, 2002

\begin{abstract}
We introduce a fiber ring optical resonator based on adiabatic fused-fiber grating couplers. The coupling of a through fiber to the resonator is controlled by the strength of the fiber Bragg gratings. By using two of these couplers and incorporating erbium-doped (ED) fiber in the ring, we control the internal loss of the ring by pumping the ED fiber. The transmission spectra of the through port and the drop port of a four-port configuration, a ring coupled to two waveguides, are measured. We show that the loss/coupling ratio of the ring-fiber system can be changed and thus that the transmission properties of the fiber can be controlled. (C) 2002 Optical Society of America

OCIS codes: $230.5750,060.1810,140.4780$.
\end{abstract}

We describe a fiber ring optical resonator in which an internal round trip involves one or more reflections from a grating coupler. In addition to the novelty of the resonator, ${ }^{1-3}$ it affords a new means of controlling coupling by varying the strength of the grating that couples the ring resonator to the through fiber. This coupling control may offer advantages compared with control schemes that depend on mechanical position control. ${ }^{4}$ Other types of fiber grating filter can be used instead of the fused-fiber adiabatic grating described here, but some of these others depend on interference ${ }^{5}$ and thus on precise dimensional control, which renders them less attractive.

The generic geometry is illustrated in Fig. 1. Each coupling region is described by a universal detailindependent $t^{6}$ matrix equation such as

$$
\left[\begin{array}{l}
a_{2} \\
C_{2}
\end{array}\right]=\left[\begin{array}{cc}
t_{a} & \kappa_{a} \\
-\kappa_{a} * & t_{a}^{*}
\end{array}\right]\left[\begin{array}{l}
a_{1} \\
C_{1}
\end{array}\right]
$$

for the coupling between ring $C$ and waveguide $a$, and similarly for the coupling between the ring and waveguide $b$. We assume that the coupling is lossless, so $|t|^{2}+|\kappa|^{2}=1$ for each coupler, and assume that all loss in the device is within the ring and is described by loss parameter $\alpha$ such that the fractional internal intensity loss per circulation is $1-\alpha^{2}$. The normalized intensity output of this device at $a_{2}$ for an input at $a_{1}$ is then ${ }^{6}$

$$
\begin{aligned}
\left|\frac{a_{2}}{a_{1}}\right|^{2} & =\frac{\alpha_{a}^{2}+\left|t_{a}\right|^{2}-2 \alpha_{a}\left|t_{a}\right| \cos \theta}{1+\alpha_{a}{ }^{2}\left|t_{a}\right|^{2}-2 \alpha_{a}\left|t_{a}\right| \cos \theta} \\
& =\left.\frac{\left(\alpha_{a}-\left|t_{a}\right|\right)^{2}}{\left(1-\alpha_{a}\left|t_{a}\right|\right)^{2}}\right|_{\theta=0,2 \pi},
\end{aligned}
$$

where we have defined a new loss parameter, $\alpha_{a}=$ $\alpha\left|t_{b}\right|$. This definition allows for the fact that, viewed from the input side, the second coupling region, which is characterized by $t_{b}$, is just another loss element in the ring with a total loss parameter $\alpha\left|t_{b}\right|$. Circulation about the ring is then described by $C_{1}=\alpha_{a} \exp (i \theta) C_{2}$, where $\theta$ is the total phase shift per circulation, including the gratings' reflections. The normalized intensity output at $b_{2}$ for an input at $a_{1}$ is

$$
\begin{aligned}
\left|\frac{b_{2}}{a_{1}}\right|^{2} & =\frac{\left(1-\left|t_{a}\right|^{2}\right)\left(1-\left|t_{b}\right|^{2}\right) \alpha}{1+\alpha^{2}\left|t_{a}\right|^{2}\left|t_{b}\right|^{2}-2 \alpha\left|t_{a}\right|\left|t_{b}\right| \cos \theta} \\
& =\left.\frac{\left(1-\left|t_{a}\right|^{2}\right)\left(1-\left|t_{b}\right|^{2}\right) \alpha}{\left(1-\alpha\left|t_{b}\right|\left|t_{a}\right|\right)^{2}}\right|_{\theta=0,2 \pi} .
\end{aligned}
$$

From Eq. (3) we see that lossless, full power switching at resonance (zero output at $a_{2}$ and all incident power leaving from $b_{2}$ ) will occur only if $\left|t_{a}\right|=\left|t_{b}\right|$ and $\alpha=1$.

For the Bragg grating coupler used in this experiment the elements of the coupling matrix in Eq. (1) $\mathrm{are}^{7}$

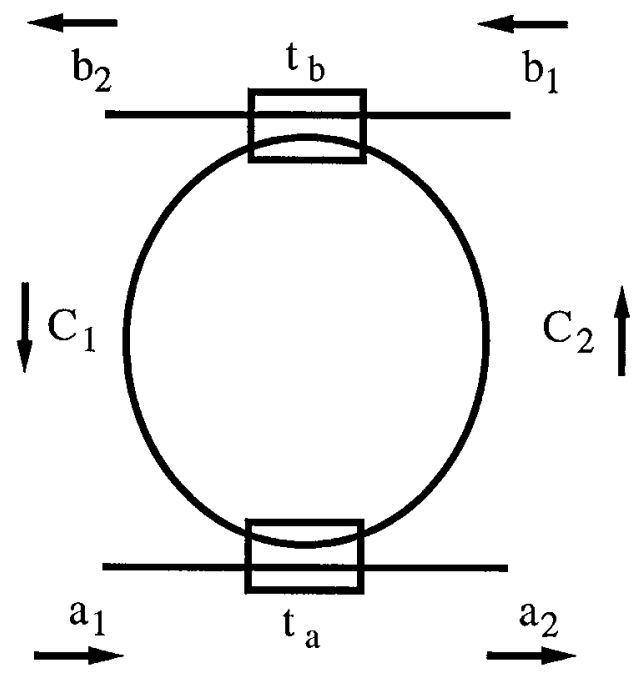

Fig. 1. Generic ring resonator $C$ coupled to waveguide $a$ and waveguide $b$. The round-trip condition that describes the circulation about the ring is $C_{1}=\alpha t_{b} \exp (i \theta) C_{2}$, where $1-\alpha^{2}$ is the propagation loss and $\theta$ is the phase delay. 


$$
\begin{aligned}
t_{a} & =\frac{-i K^{*} \sinh (\Gamma L)}{\Gamma \cosh (\Gamma L)+i(\Delta \beta / 2) \sinh (\Gamma L)} \\
\kappa_{a} & =\frac{\Gamma \quad(\text { grating reflectance }),}{\Gamma \cosh (\Gamma L)+i(\Delta \beta / 2) \sinh (\Gamma L)} \\
\Gamma^{2} & =|K|^{2}-\left(\frac{\Delta \beta}{2}\right)^{2}, \quad \Delta \beta=2 \beta-\frac{2 \pi}{\Lambda} \\
K & \approx \frac{\omega}{c} \Delta n_{\text {grating }} .
\end{aligned}
$$

In this case, one can control coupling matrix elements $\kappa_{a}$ and $t_{a}$ by varying grating strength $K$ and (or) frequency $\omega$.

From Eq. (2) we define the critical coupling condition at resonance as one for which all the power is transferred from $a_{1}$ to the ring, i.e., $\left|a_{2}\right|^{2}=0$. This condition occurs when $\alpha_{a}=\left|t_{a}\right|$. By controlling the losses in the ring, i.e., controlling $\alpha$, we may affect the coupling to the ring and thus the transmission properties of the through fiber. For sufficiently small values of coupling, $\left|t_{a}\right| \sim 1$, the transmission is highly sensitive to small changes in $\alpha_{a}$ in the overcoupled regime, $\alpha_{a}>\left|t_{a}\right|$, as can be seen from Fig. 2(a). Although it is not always convenient to work in this regime because of the intrinsic losses in most materials, for an all-fiber device we take advantage of erbium-doped (ED) fiber to provide gain and achieve the overcoupled condition.

A schematic of our device is shown in Fig. 3. The interaction mechanism was provided by two adiabatic fused-fiber grating couplers. ${ }^{8}$ Without the gratings, light entering as $a_{1}$ will continue, essentially, without loss to arm $C_{2}$ and exit as $b_{2}$. Similarly, light entering as $b_{1}$ will travel along arm $C_{1}$ and exit at $a_{2}$. Introducing the grating causes wavelengths near the Bragg value, $\lambda_{\text {Bragg }}$, to see the gratings. For incident wave $a_{1}$, a wave $t_{a} a_{1}$ proceeds to $a_{2}$ while $\kappa_{a} a_{1}$ joins $C_{2}$. Coefficients $t_{a}$ and $\kappa_{a}$ are given, respectively, by Eqs. (4) and (5).

Employing two of these adiabatic couplers, as shown in Fig. 3, causes a ring to be formed by arms $C_{1}$ and $C_{2}$. This ring is apparent only to wavelengths near $\lambda_{\text {Bragg, }}$ and these see the same generic geometry as Fig. 1 with complex grating reflectances $r_{a}$ and $r_{b}$ assuming formally the roles of parameters $t_{a}$ and $t_{b}$ as given by Eq. (4). Thus, control of the coupling, which is the key for all applications of such resonators, is achieved by control of the gratings' strength. This control is particularly important for the matched couplers needed for full power switching. An ED fiber was incorporated into arm $C_{1}$ and pumped with a 980-nm laser through ports $b_{1}$ and $a_{2}$, allowing us to control $\alpha$ by controlling the pump power. Splicing the ED fiber into arm $C_{1}$ rather than into arm $C_{2}$ causes nonresonant wavelengths to pass through the device unaffected by the erbium; only the resonant wavelength will be affected by the ring resonances.

The measured transmission of the through and drop ports at resonance, as shown in Fig. 3, are shown in Figs. 2(a) and 2(b), respectively, as functions of $\alpha$, the loss parameter. To achieve the wide range of $\alpha$ we scanned the current in pump 2 from 50 to $350 \mathrm{~mA}$ with pump 1 off and then turned on pump 1 and scanned pump 2 again. The measured intensity from pump 2 ranged from 3.17 to $13.48 \mathrm{dBm}$, and the measured intensity of pump 1 was $18.65 \mathrm{dBm}$. From the transmission spectra showing normalized transmission as a function of the detuning from resonance in Fig. 4 we inferred $\alpha$ by fitting Eqs. (2) and (3) to the linewidth and the depth of the resonance. From these spectral measurements we determined that the bandwidth was $\sim 5 \mathrm{MHz}$ and the free spectral range was $150 \mathrm{MHz}$ because of the length of the ring, $1.5 \mathrm{~m}$. Within the ring, arm $C_{1}$ consisted of $51 \mathrm{~cm}$ of high-concentration ED fiber. The coupling values were measured to be $\left|t_{a}\right|=\left|r_{a}\right|=0.856$ and $\left|t_{b}\right|=\left|r_{b}\right|=0.991$. The highly mismatched coupling of our device resulted in small signals from the drop port, $b_{2}$. However, from the measurements taken of the through port, $\alpha_{2}$, we observed the main feature required of an efficient switch or modulator, a high degree of sensitivity to the control parameter. From Fig. 4(a) we see that, near critical coupling, $\alpha=0.910$, we achieve nearly $-16.2-\mathrm{dB}$ extinction. When the resonator is

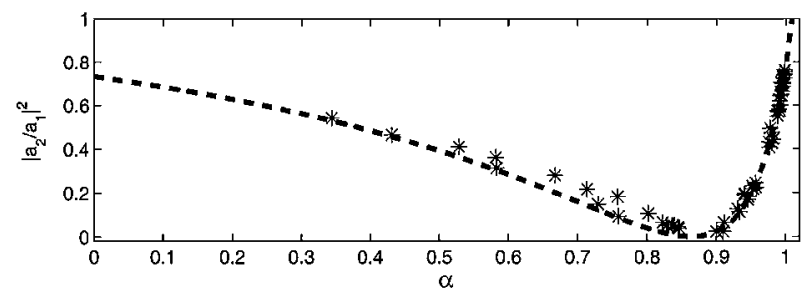

(a)

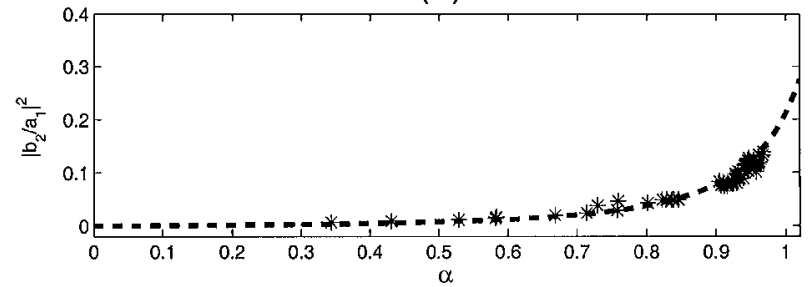

(b)

Fig. 2. Normalized transmission of the device at resonance as we scan $\alpha$ by controlling the pumping of the ED fiber. (a) The through port, $a_{2}$. In the overcoupled region, $\alpha>t_{a} / t_{b}$, we see that there is very sensitive control of the transmission. (b) The drop port, $b_{2}$.

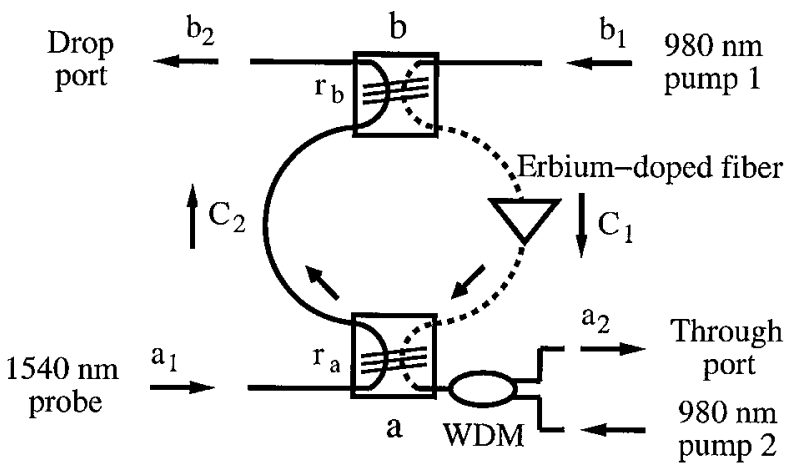

Fig. 3. Schematic of the experimental setup. $\left|r_{a}\right|=\left|t_{a}\right|=$ 0.856 and $\left|r_{b}\right|=\left|t_{b}\right|=0.991$. ED fiber was spliced into ring $C$ to provide control of loss parameter $\alpha$. 


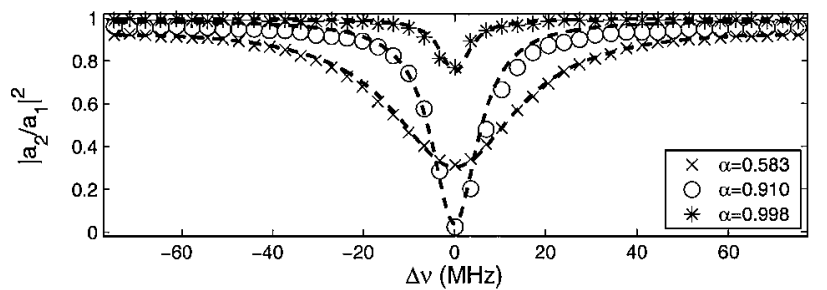

(a)

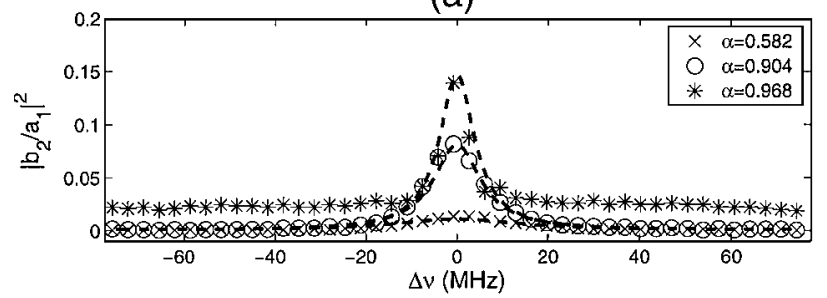

(b)

Fig. 4. Normalized transmission as a function of detuning from resonance in optical frequencies. (a) The through port, $a_{2}$. The three curves shown are representative of the undercoupled $\left(\alpha<t_{a} / t_{b}\right)$, the critically coupled $\left(\alpha=t_{a} / t_{b}\right)$, and the overcoupled $\left(\alpha>t_{a} / t_{b}\right)$ regions. (b) The drop port, $b_{2}$, at similar values of $\alpha$.

overcoupled, $\alpha=0.998$, we achieve approximately $-1.18 \mathrm{~dB}$ of transmission loss. This translates to a 15-dB signal modulation by use of a small change in $\alpha$, $\Delta \alpha=0.0879$, with an insertion loss of approximately $1 \mathrm{~dB}$. However, because of the saturation effects of the ED fiber, this change in $\alpha$ required pump powers from 11.29 to $27.85 \mathrm{dBm}, \Delta P_{\text {pump }}=16.56 \mathrm{dBm}$. In addition, the switching-modulation time of the device is ultimately limited by the relatively long transition rates of the erbium energy levels (approximately $10 \mu \mathrm{s}-10 \mathrm{~ms}){ }^{9}$
In conclusion, we have demonstrated a new type of optical fiber ring resonator based on asymmetric, adiabatic fused-fiber grating couplers. We achieve the control of the coupling that is essential to communication and scientific applications by varying the grating strength.

The authors thank Anthony S. Kewitsch and George Rakuljic of Arroyo Optics, Inc., for supplying the adiabatic fused-fiber grating couplers. We acknowledge the generous support of the U.S. Air Force Office of Naval Research, and the Defense Advanced Research Projects Agency. J. Choi (johnchoi@ caltech.edu) thanks the U.S. Department of Defense and the American Society for Engineering Education.

\section{References}

1. B. Crosignani, A. Yariv, and P. Di Porto, Opt. Lett. 11, 251 (1986).

2. B. Crosignani, B. Daino, P. Di Porto, and S. Wabnitz, Opt. Commun. 59, 309 (1986).

3. J. M. Choi, R. K. Lee, and A. Yariv, Opt. Lett. 26, 1236 (2001).

4. L. F. Stokes, M. Chodorow, and H. J. Shaw, Opt. Lett. 7, 288 (1982).

5. F. Bilodeau, D. C. Johnson, S. Thériault, B. Malo, J. Albert, and K. O. Hill, IEEE Photon. Technol. Lett. 7, 388 (1995).

6. A. Yariv, Electron. Lett. 36, 32 (2000).

7. A. Yariv and P. Yeh, Optical Waves in Crystals (Wiley, New York, 1984), p. 188.

8. A. S. Kewitsch, G. A. Rakuljic, P. A. Willems, and A. Yariv, Opt. Lett. 23, 106 (1998).

9. E. Desurvire, Erbium-Doped Fiber Amplifiers (Wiley, New York, 1994). 